\title{
A Survey of Emergency Communication Network Architectures
}

\author{
D. Srinivasa rao ${ }^{1}$, P. Devi Pradeep ${ }^{2}$ and B. Anil Kumar ${ }^{3}$ \\ ${ }^{1,2,3}$ Asst.professor, Dept. of ECE, GMR Institute of Technology, Rajam, India \\ ${ }^{1}$ srinivasarao.d@gmrit.org, ${ }^{2}$ devipradeep.p@gmrit.org, ${ }^{3}$ anilkumar.b@gmrit.org
}

\begin{abstract}
The recent severe cyclone storm in India named 'Hudhud' was considered as the second strongest tropical cyclone of 2014 within the North Indian Ocean. The entire communication network is lost and power lines were disrupted for three north eastern districts of Andhra Pradesh, India. It took several days to resume the communication services. This lack of robust network connection has become one of the major topics for any discussion of a Disaster Information Network System. Hence, there is huge demand to establish a robust communication network which never fails during emergency times. In this paper, a survey of emergency communication network architectures has been done in detail. The existing network architectures have got several practical issues which need to be addressed. We also highlight the implementation issues of emergency communication networks which may be considered as scope of this topic.
\end{abstract}

Keywords: cyclone, disaster, base station, network architecture, MANET

\section{Introduction}

There have been a frequent number of earthquakes, tsunamis, cyclones and floods from time to time across the entire globe. Recent examples include the earthquake on Haiti in 2010, the Japanese earthquake and tsunami in 2011, the Chilean earthquake in 2011 and the Hurricane Sandy in USA in 2012.Now, India is one among the most disaster prone regions in the world. It also experienced some of the world's most severe droughts, famines, cyclones, earthquakes, chemical disasters, mid-air head-on air collisions, rail accidents, and road accidents. After the 2004 Indian Ocean tsunami, India started the disaster management system and there has been a paradigm shift to become more proactive with emphasis on disaster prevention, mitigation and preparedness. Although India is contributing much in preventing loss of life through effective pre-disaster warning system, the communication networks are been severely damaged due to lack of robustness. The collapse of communications infrastructure is the usual effect of disaster, due to the collapse of antennas, buildings, power etc. There are many lessons to be learnt in disaster management and environment rules in the wake of 'Hudhud' cyclone which caused devastation in Vishakhapatnam, India. Though evacuation was much successful, other aspect of management like communication network failure, power supply disruptions are need to be addressed. Therefore, a number of standardization activities and research efforts have been started quite recently in the area of disaster-resilient [1] communication.

Cellular Mobile Networks are the dominant technology in the today's communication world. In the recent statistics from ITU, there are almost 7 billion mobile-cellular subscriptions worldwide. These are likely to get disturbed easily when a natural disaster (earthquake, tsunami, flooding, etc.) hits an area because of their centralized control and high-power requirements. While the need for communication and other types of ICT services is very high after such events, the communication infrastructure is often damaged to large extents, making services unavailable or at least heavily congested. 
This paper discusses a brief survey of communication networks architecture for emergency and is organized as follows: Section 2 discusses the brief scenario of emergency communication during disaster [2]. Section 3 highlights the existing mobile network architectures and technologies for emergency management. It presents in brief the main modern wireless and cellular networks used in emergency situations. Section 4 constitutes the major issues to be considered while developing modern mobile network architectures. Finally, Section 5 concludes the paper.

\section{Emergency Communication System Illustration}

\subsection{Pre Disaster Communications}

Prior to a disaster, the main function of emergency communications is disaster warning. The outline of main steps in the disaster warning process [2] is given below.

Step1: Sensors employed in the field transmit local environmental information at regular intervals to the data processing center. For example, seismic sensors transmit earthquake related information that can be used for warnings about earthquakes and/or tsunamis. The communications can be done via wireline, wireless, or satellite transmission. Figure 1 illustrates communications from sensors to the data processing center.

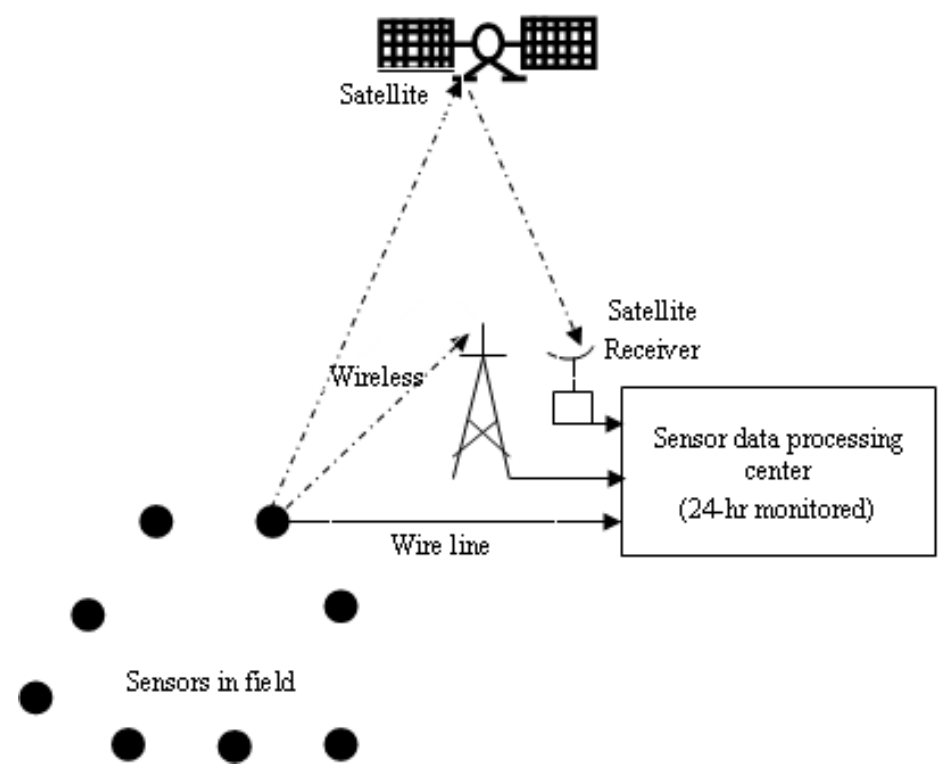

Figure. 1. Data Transmission from Sensors to the Processing Center

Step2: The sensor data processing center, assumed here is monitored by humans 24 hours a day, analyzes the sensor data and makes a decision whether or not disaster warnings should be issued. Note that this decision is done by humans and not automated.

Step3: If the decision is to issue warnings, then the warning information will be distributed with no human involvement beyond the data processing center. The warnings can reach the individuals in the disaster area via wireless or wireline transmission, or by the use of visual/sound alarms. Fig. 2 illustrates communications from the data processing center to individuals in disaster related areas. 


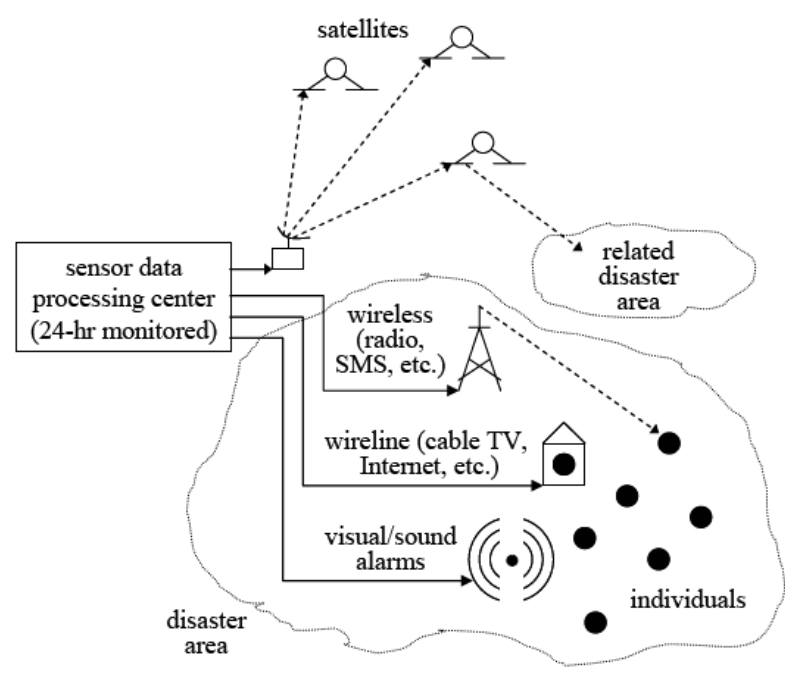

Figure. 2. Transmission of Disaster Warnings

The technical contributions involve how the warnings can be distributed through existing communication infrastructure with only slight modifications of the existing systems, and with no human intervention required. For example, if the warnings are distributed using SMS messages in cellular systems, we may be able to use the control channels as communication channels in case of emergency.

\subsection{Post Disaster Communications}

After the disaster such as an earthquake or tsunamis, we expect that some or all communication infrastructure in the disaster related areas would be destroyed. The main function of emergency communications [3] is to set up communication networks so that survivors can establish a contact or can be located by the rescue teams through common electronic devices such as cellular phones.

\section{Mobile Network Architectures}

In this section, we outline three possible strategies for setting up emergency communication networks.

\subsection{Emergency Network Based on LDRU:}

In the case that some base stations of cellular networks remain after the disaster, we can utilize the remaining base stations. However, for emergency communications, we will need to change the transmission scheme (e.g. different frequency band, higher power, etc.) for larger coverage areas. In addition, a locally deployed resource unit [3] may be equipped with a radio base station that can also be used to set up the emergency network. For emergency communications, the mobile phone units must be able to operate in the emergency mode with a compatible transmission scheme. Figure 3 illustrates emergency communications based on surviving base stations from the cellular phone systems. 


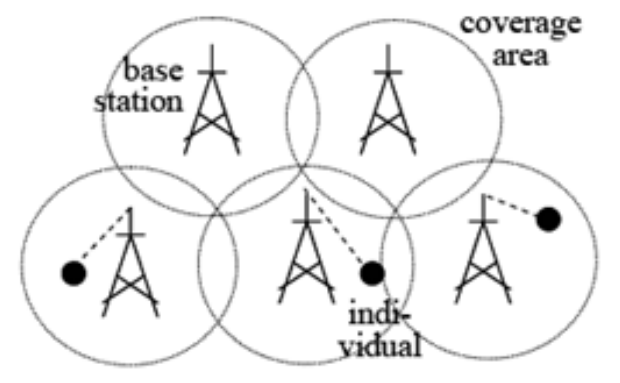

a) Before disaster

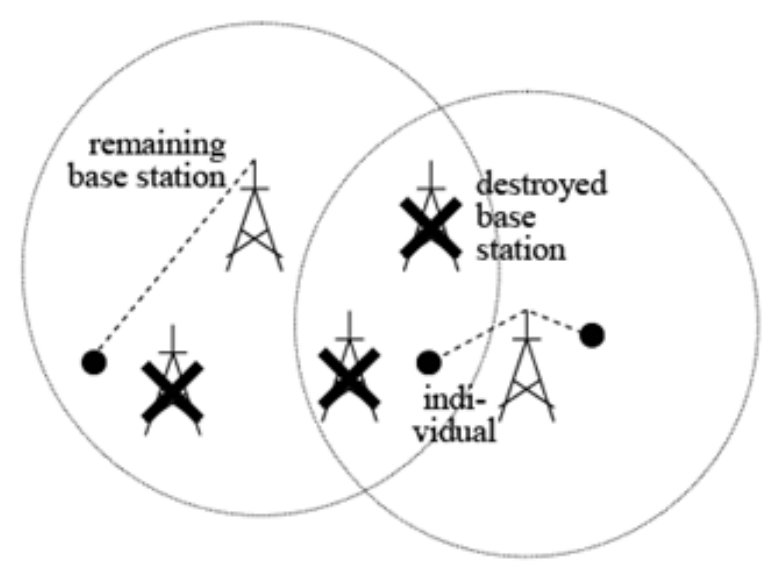

b) after disaster

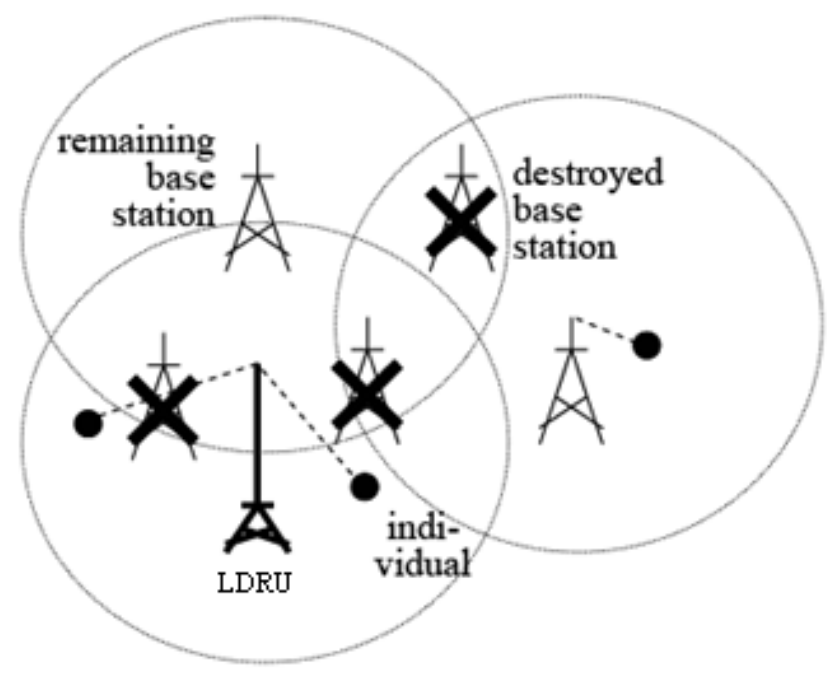

C) After the disaster with LDRU

\section{Figure 3. Emergency Network based on the Remaining Cellular Base Stations as Well as LDRU (Locally Deployed Resource Unit), if any}

\subsection{Emergency Network based on Satellite:}

The above strategy based on using the remaining base stations may not be applicable when all base stations are destroyed. In this case, one possibility is to use satellite communications for each survivor. For this scheme to be possible, a regular mobile phone needs to be able to communicate with a satellite. The modifications of existing systems may include having high power emergency channels available in the satellite, add-on extendable antennas for the mobile phone, and hardware/software reconfiguration in the mobile units, and so on. Fig. 4 illustrates emergency communications based on a direct contact between each survivor and a satellite. 


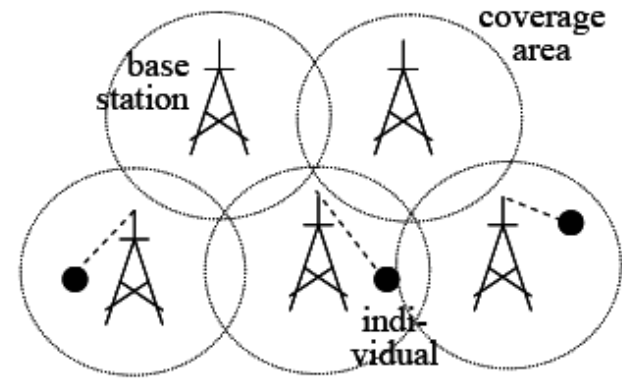

a) before disaster

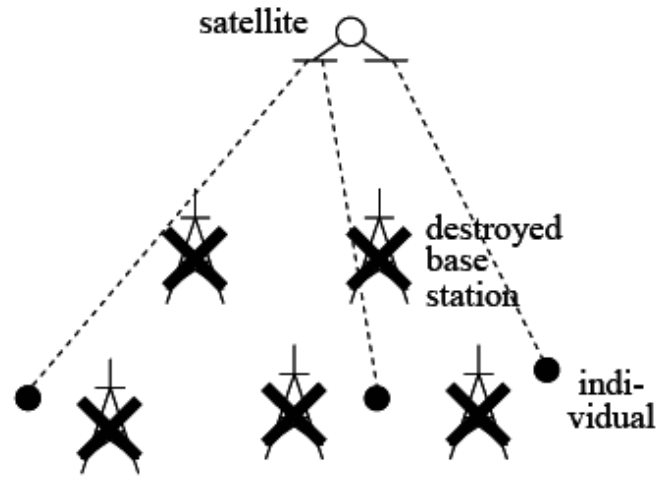

b) after disaster

Figure 4. Emergency Network based on Direct Satellite Communications

\subsection{Emergency Network based on Adhoc-network}

In the cases where adding the satellite feature to each regular mobile phone according to Figure 4 is too expensive, we can consider an alternative network architecture. In this architecture, a small subset of local users, e.g. police and rescue officers, carry mobile phones that are satellite capable. The other mobile phones are modified so that they can communicate with these satellite capable phones, forming an ad-hoc network [5] when the base stations are all destroyed. Figure 5 illustrates emergency communications based on the use of ad-hoc networks.

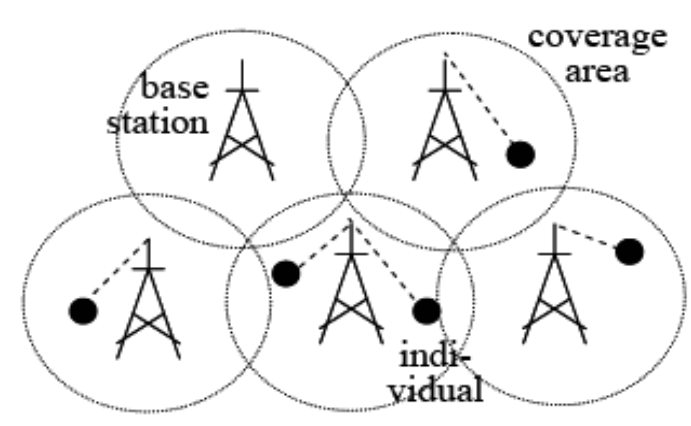

(a) before the disaster

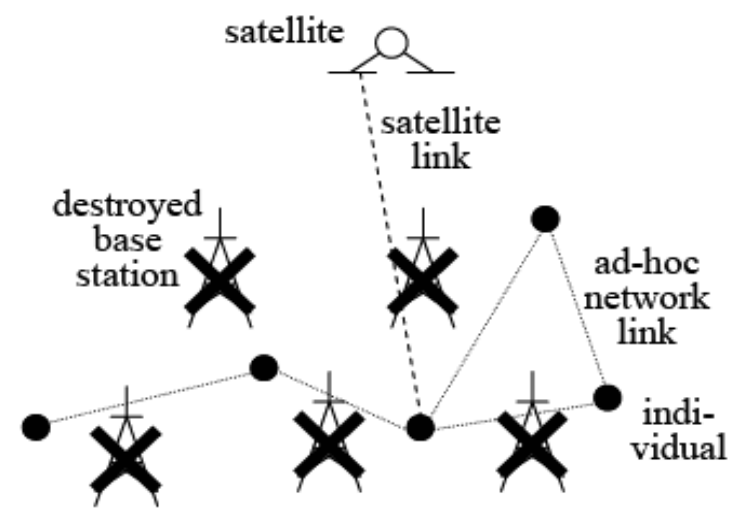

(b) after the disaster

Figure 5. Emergency Network based on an Ad-hoc Network

\section{Issues Under Consideration}

\subsection{Sensors}

Sensors play vital role in Pre-disaster communications. Sensors employed in the field transmit local environmental information at regular intervals of time to an emergency operating center for data processing. For example, seismic sensors transmit earthquake related information that can be used for warnings about earthquakes and/or tsunamis. The communications can be done via wire-line, wireless, or satellite transmission. Hence, lot of care must be taken in choosing sensor nodes for intended application. They have to fulfill quite different requirements regarding battery life, mechanical robustness of the node's housing, size, and so on. Since nodes may run out of energy or might be damaged, 
or since the wireless communication between two nodes can be permanently interrupted, it is important that the sensor network as a whole is able to tolerate such faults. To tolerate node failure, redundant deployment is necessary, using more nodes than would be strictly necessary if all nodes functioned correctly.

In many scenarios, nodes should have to rely on a limited supply of energy (using batteries). Replacing these energy sources in the field is usually not practicable, and simultaneously, a sensor node must operate at least for a given mission time or as long as possible. Hence, the lifetime of a sensor node becomes a very important figure of merit. Evidently, an energy-efficient way of operation of the sensor is necessary. Therefore building a pre-disaster emergency network [11] first of all requires the constituting sensor nodes to be developed and available.

\subsection{Network Architectures}

The current evolution in cellular radio access networks is to migrate from a centralized architecture to a distributed architecture. This trend is driven by the demand for everincreasing data rates, resulting in numerous cell sites and ever-smaller cell sizes. One of the main objectives of this architectural evolution is to harness the benefits of the core Internet Protocol (IP) philosophy while maintaining the benefits of proven air interface technologies for wide area network deployments. Moving the network intelligence to the edge of the network leads to performance gains by making use of the knowledge and awareness of the wireless channel. In addition, consolidating the radio access network functionalities increases network efficiency by decreasing transmission and processing delays in the radio access network, thereby achieving greater network capacity [10].

\subsection{Communication Link Establishment}

Establishing a communication link is a major enabler for the management of humanitarian aid and emergency response operations in any disaster event. But when needed the most terrestrial and cellular networks can be among the first causalities of a disaster. Therefore, we have to look beyond all these systems to find alternative mode of communication systems aiming to provide simple, strong, reliable and disaster resilient communication links in emergency situations.

\subsection{Low Power Coding and Modulation}

As the entire power line communication would be disrupted, power becomes a scarce resource during disaster times. Hence the power must be utilized very effectively at both base stations and mobile users. The signal processing blocks will consume a major amount of power at the base stations. This can be minimized only with power efficient modulation and coding schemes [12]. There is a need to overcome several tradeoffs to develop such robust techniques.

\subsection{Mobile Ad-hoc Networks}

Mobile ad-hoc networks are infrastructure-less networks since they do not require any fixed infrastructure, such as a base station, for their operation. Mobile devices rely on batteries for energy. Battery power is finite, and represents one of the greatest constraints in designing algorithms for mobile devices. Projections on progress in battery technology show that only small improvements in the battery capacity are expected in next future. Under these conditions, it is vital that power utilization be managed efficiently by identifying ways to use less power, preferably with no impact on the applications. Limitation on battery life, and the additional energy requirements for supporting network operations (e.g., routing) inside each node, make the energy conservation one of the main concern in ad hoc networking. Another major issue in ad-hoc networks [8] is their limited 
transmission range. Each node will be able to communicate directly with any other node that resides within its transmission range. For communicating with nodes that reside beyond this range, the node needs to use intermediate nodes to relay the messages hop by hop. The specific MANET issues and constraints described above pose significant challenges in ad-hoc network design. A large body of research has been accumulated to address these specific issues, and constraints.

\section{Conclusion}

Natural disasters are of increasing frequency and severity in the modern world. For the past years wireless technology has seen a tremendous growth in communications. To provide the command, control and communications capabilities needed in emergency situations, public safety and emergency management organizations increasingly rely on wireless technology. However, survival of existing wireless mobile networks is very difficult during emergency situations especially in the sea coast areas which are easily prone to cyclones and tsunamis. Hence there is a need to build robust communication network to provide services at those extreme conditions. This paper presents a survey of various mobile network architectures for emergency response communications. The proposed schemes have got some issues which need to be considered for effective communication during disaster times. These issues are driving new approaches to provide effective solutions during emergency situations.

\section{References}

[1] ITU-T Focus Group on Disaster Relief Systems (FG-DR\&NRR), Network Resiliency and Recover, http://www.itu.int/en/ITU-T/focusgroups/drnrr

[2] N. Rajatheva, D. Gamage, K. Ahmed and P. Saengudomlert, "Emergency Communications during Natural Disasters: Infrastructure and Technology", Asian Institute of Technology,Bangkok, Thailand, (2005).

[3] T. Sakano, Z. Md. Fadlullah, T. Ngo, H. Nishiyama, M. Nakazawa, F. Adachi, N. Kato, A. Takahara, T. Kumagai, H. Kasahara and S. Kurihara, "Disaster-Resilient Networking: A New Vision Based on Movable and Deployable Resource Units", IEEE Network Magazine, vol. 27, no. 4, July-August (2013), pp. 40-46.

[4] Y.-T. Chiu, "Japan Looks to Taiwan to Disaster-Proof Telecom”, IEEESpectrum, (2013) February.

[5] Z. Shao, Y. Liu, Y. Wu and L. Shen, "A Rapid and Reliable Disaster Emergency Mobile Communication System via Aerial Ad Hoc BS Networks", in Proc of IEEE Wireless Communications, Networking and Mobile Computing (WiCOM), (2011) September.

[6] N. Marchetti, "Telecommunications in Disaster areas", River publishers, (2010).

[7] Y. Bai, W. Du, Z. Ma, C. Shen, Y. Zhou, and B. Chen, "Emergency communication system by heterogeneous wireless networking", in Proc. of IEEE Wireless Communications, Networking and Information Security (WCNIS), (2010) June.

[8] Y.-N. Lien, H.-C. Jang, and T.-C. Tsai, "A manet based emergency communication and information system for catastrophic natural disasters", In Proceedings of the 29th IEEE International Conference on Distributed Computing Systems Workshops, (2009).

[9] W. Kuji,G. Satou, K. Koide, Y. Shibata and N. Shiratori, "Never-Die Network and Disaster-Control System", Jane 2008. IPSJ SIG-DPS: Proceedings of information Processing Society of Japan, (2008), pp. 131-135.

[10] W. C. Y. Lee, "Mobile Cellular Telecommunications", McGraw-Hill, New York, New York, USA, (2006).

[11] K. Lorincz, "Sensor Networks for Emergency Response: Challenges and Opportunities", Pervasive Computing, (2004).

[12] B. Sklar, "Digital Communications", Fundamentals and Applications Pearson Education Asia, (2001). 


\section{Authors}

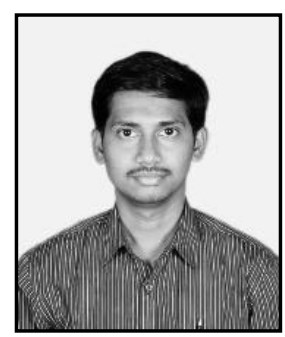

D. Srinivasa rao, he did Masters in Engineering (Communication systems) from Thiagarajar College of Engineering, Madurai. Now, he is pursuing $\mathrm{PhD}$ from VIT Univeristy, Chennai. He got nearly five years' experience in teaching. He is currently working as Asst.professor in Dept. of ECE, GMR Institute of Technology, Rajam, India. His research interests include Wireless communications, Next generation networks, and Coding techniques.

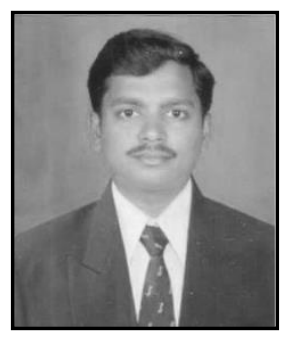

P. Devi Pradeep, he received M.Tech degree from National Institute of Technology, Warangal, Andhra Pradesh, India. B.Tech degree in Electronics and Communication Engineering from Vitam College of engineering affiliated to JNTU Hyderabad, Andhra Pradesh, India. He is joined as Assistant Professor in the Department. Of Electronics and Communication Engineering at GMR Institute of Technology, Rajam, Srikakulam District, Andhra Pradesh, India in 2009.Having Total teaching experience is 9 years. His research interests are Video Processing, Analog and digital VLSI Design. He is a life member of ISTE Since 2009. 